\title{
CONNECTIVITY-PRESERVING TRANSFORMATIONS OF BINARY IMAGES
}

\author{
Prosenjit Bose $^{*} \quad$ Vida Dujmović ${ }^{*} \quad$ Ferran Hurtado $^{\dagger} \quad$ Pat Morin *
}

ABSTRACT. A binary image $I$ is $B_{a}, W_{b}$-connected, where $a, b \in\{4,8\}$, if its foreground is $a$-connected and its background is $b$-connected. We consider a local modification of a $\mathrm{B}_{a}, \mathrm{~W}_{b}$-connected image $I$ in which a black pixel can be interchanged with an adjacent white pixel provided that this preserves the connectivity of both the foreground and the background of $I$. We have shown that for any $(a, b) \in\{(4,8),(8,4),(8,8)\}$, any two $\mathrm{B}_{a}, \mathrm{~W}_{b}$-connected images $I$ and $J$ each with $n$ black pixels differ by a sequence of $\Theta\left(n^{2}\right)$ interchanges. We have also shown that any two $\mathrm{B}_{4}, \mathrm{~W}_{4}$-connected images $I$ and $J$ each with $n$ black pixels differ by a sequence of $O\left(n^{4}\right)$ interchanges.

\section{Introduction}

We call a function $I: \mathbb{Z}^{2} \rightarrow\{0,1\}$ a binary image. We call the elements of $\mathbb{Z}^{2}$ pixels and we say that a pixel $p$ is black (respectively, white) if $I(p)=1$ (respectively, $I(p)=0$ ). We say that a binary image is finite if it has a finite number of black pixels. We only consider finite binary images in this paper.

Let $G_{4}$ be the graph whose vertex set is $\mathbb{Z}^{2}$ (the set of all pixels) and in which two pixels $\left(x_{1}, y_{1}\right)$ and $\left(x_{2}, y_{2}\right)$ are adjacent if and only if $\left(x_{1}-x_{2}\right)^{2}+\left(y_{1}-y_{2}\right)^{2}=1$, that is, $G_{4}$ is the integer lattice. The graph $G_{8}$ is the graph whose vertex set is $\mathbb{Z}^{2}$ and in which two pixels $\left(x_{1}, y_{1}\right)$ and $\left(x_{2}, y_{2}\right)$ are adjacent if and only if $\left(x_{1}-x_{2}\right)^{2}+\left(y_{1}-y_{2}\right)^{2} \leq 2$, that is, $G_{8}$ is the integer lattice in which two diagonals have been added to every face. Two pixels are 4-neighbours (respectively, 8-neighbours) if they are adjacent in $G_{4}$ (respectively, $G_{8}$ ). Given a binary image $I$, the graph $B_{4}(I)$ (respectively, $B_{8}(I)$ ) is the subgraph of $G_{4}$ (respectively, $G_{8}$ ) induced by the black pixels in $I$ and the graph $W_{4}(I)$ (respectively, $W_{8}(I)$ ) is the subgraph of $G_{4}$ (respectively, $G_{8}$ ) induced by the white pixels (see Figure 1). For $a, b \in\{4,8\}$ we say that an image $I$ is $B_{a}, W_{b}$-connected if the graphs $B_{a}(I)$ and $W_{b}(I)$ are each connected, that is, each has a single connected component. Note that a binary image $I$ is $B_{a} W_{8}$-connected, $a \in\{4,8\}$, if and only if $B_{a}(I)$ is connected and $B_{4}(I)$ does not contain a cycle $C$ such that in $I$ there is a white pixel inside $C$. Similarly, a binary image $I$ is $B_{a} W_{4}$-connected, $a \in\{4,8\}$, if and only if $B_{a}(I)$ is connected and $B_{8}(I)$ does not contain a cycle $C$ such that in $I$ there is a white pixel inside $C$.

In this paper we consider a local modification operation on binary images in which a black pixel $p$ and a white pixel $q$ are interchanged, that is their colours are interchanged. More precisely, we perform the interchange $\langle p, q\rangle$ on $I$ to obtain the image $I^{\prime}$ where

$$
I^{\prime}(x)= \begin{cases}I(p) & \text { if } x=q \\ I(q) & \text { if } x=p \\ I(x) & \text { otherwise }\end{cases}
$$

We say that the interchange $\langle p, q\rangle$ is 4-local (respectively, 8-local) if $p$ and $q$ are adjacent in $G_{4}$ (respectively, $G_{8}$ ). In this paper we are primarily concerned with 8-local interchanges and we are interested

\footnotetext{
*School of Computer Science, Carleton University, Ottawa, Canada. Email: \{jit, morin, vida\}@scs . carleton. ca. Research partially supported by NSERC.

†Departament de Matemàtica Aplicada II, Universitat Politècnica de Catalunya (UPC), Barcelona, Spain. Email: Ferran.Hurtado@upc.edu. Research partially supported by Projects MCYT-FEDER BFM2003-00368 and Gen. Cat 2001SGR00224.
} 




(a)

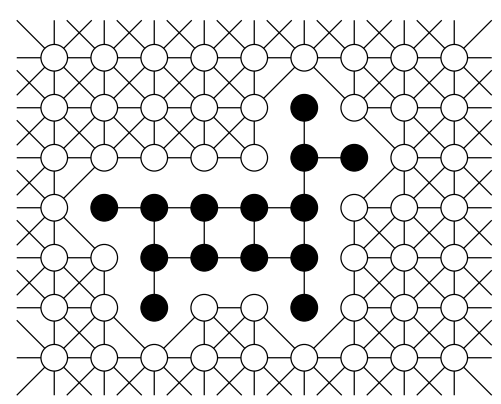

(b)

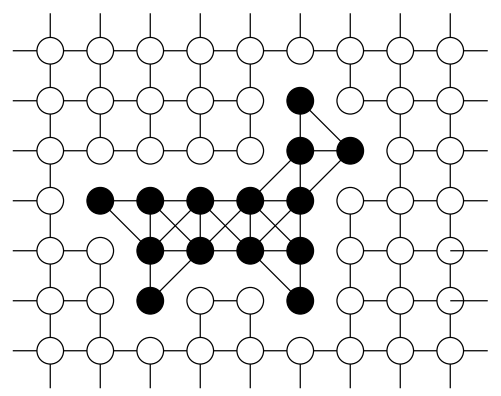

(c)

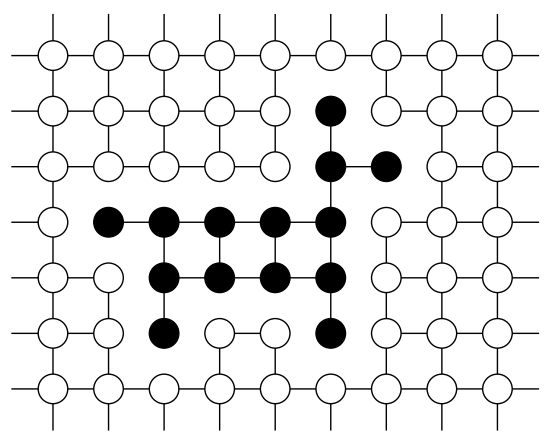

(d)

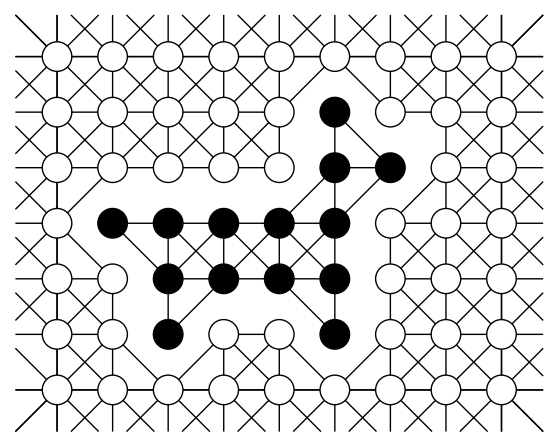

(e)

Figure 1: (a) a binary image $I$, (b) the graphs $B_{4}(I)$ and $W_{8}(I)$, (c) the graphs $B_{8}(I)$ and $W_{4}(I)$, (d) the graphs $B_{4}(I)$ and $W_{4}(I)$, and (e) the graphs $B_{8}(I)$ and $W_{8}(I)$.

in whether two images with the same number of black pixels differ by a sequence of connectivity-preserving interchanges. More precisely, we say that two $\mathrm{B}_{a}, \mathrm{~W}_{b}$-connected images $I$ and $J$ are $(a, b)$-IP-equivalent [8] if there exists a sequence of images $I_{0}=I, I_{1}, \ldots, I_{r}=J$ such that each $I_{i}$ is $\mathrm{B}_{a}, \mathrm{~W}_{b}$-connected and $I_{i}$ can be converted into $I_{i+1}$ by a single (8-local) interchange.

\subsection{Previous Work}

The study of connectivity in digital images seems to have been first initiated by Rosenfeld $[4,5,6]$ and has since become part of the field of digital topology [2,3]. The idea of using connectivity-preserving interchanges (IP-equivalence) to convert one image into another appears in a sequence of papers by Rosenfeld et al $[7,8,9]$.

Rosenfeld, Saha, and Nakamura [9] study interchanges and (among other things) show that any two $\mathrm{B}_{4}, \mathrm{~W}_{8}$-connected digital arcs* with the same number of black pixels are $(4,8)$-IP-equivalent. The same authors conjectured that any two $\mathrm{B}_{4}, \mathrm{~W}_{8}$-connected images are $(4,8)$-IP-equivalent.

Rosenfeld and Nakamura [8] later resolved this conjecture in the affirmative by giving an algorithm for computing a sequence of 8-local interchanges to convert any $\mathrm{B}_{4}, \mathrm{~W}_{8}$-connected image $I$ with $n$ black pixels into any other $\mathrm{B}_{4}, \mathrm{~W}_{8}$-connected image $J$ with $n$ black pixels. Their algorithm achieves this by scanning $I$ with a horizontal line from top to bottom and performing interchanges while maintaining the invariant that the part of the image above the scan line consist of a set of disjoint vertical line segments. As the scan line advances, the line segments above the scan line are moved and/or merged in order to preserve this invariant. Although the authors are not concerned with the number of interchanges required to perform this conversion, examining their algorithm reveals that the number of interchanges is bounded by $O\left(n^{3}\right)$ and there exists examples for which their algorithm performs $\Omega\left(n^{3}\right)$ interchanges.

\footnotetext{
${ }^{*}$ An image $I$ is a digital arc if the graph $B_{4}(I)$ is a path.
} 
Motivated by applications in robotics, and apparently unaware of Ref. [8], Dumitrescu and Pach [1] consider the problem of converting one image into another while preserving connectivity of the graph $B_{4}$ only. Thus their definition of connectivity is weaker than that used here, however, their definition of interchange is more restricted. They show that any image $I$ for which $B_{4}(I)$ is connected can be converted into any image $J$ for which $B_{4}(J)$ is connected using a sequence of $O\left(n^{2}\right)$ 8-local interchanges that preserve connectivity of the graph $B_{4}$. They achieve this result by collecting all black pixels on a line segment. To add a new black pixel to the line segment they select a very particular pixel and move it around the boundary of the black pixels until it lies on the line segment.

\subsection{New Results}

In this paper we prove that, for any $(a, b) \in\{(4,4),(4,8),(8,4),(8,8)\}$, any two $\mathrm{B}_{a}, \mathrm{~W}_{b}$-connected images $I$ and $J$ each with $n$ black pixels are $(a, b)$-IP-equivalent. Moreover, one can be converted into the other with a sequence of $O\left(n^{2}\right)$ 8-local interchanges if $(a, b) \in\{(4,8),(8,4),(8,8)\}$ and $O\left(n^{4}\right)$ 8-local interchanges if $(a, b)=(4,4)$. To the best of our knowledge, these are the first results on $(4,4),(8,4)$ - and $(8,8)$-IPequivalence. This is also the first quadratic bound on the number of interchanges used to show $(4,8)$-IPequivalence to two arbitrary $\mathrm{B}_{4}, \mathrm{~W}_{8}$-connected images. The quadratic bounds are optimal up to constant factors since it is easy to see that converting a horizontal line segment into a vertical line segment requires $\Omega\left(n^{2}\right)$ 8-local interchanges.

It is also worth noting that our proof technique, and resulting algorithms, are of a different style than those used by Rosenfeld and Nakamura [8] and Dumitrescu and Pach [1]. For $(a, b) \in\{(4,8),(8,4),(8,8)\}$, we obtain our results by showing that, as long as $I$ is not a vertical segment, there is always a set of at most 4 black pixels that can move one by one such that the resulting image is more to the "left" or "upwards" than the one we started with. By repeatedly performing this sequence of at most four 8-local interchanges the image organizes itself into a vertical line segment. This is unlike previous algorithms $[1,8]$ in that the entire process takes place without any long-term planning about the movement of a pixel or group of pixels. The $(a, b) \in\{(4,4)\}$ version of the problem appears to be quite different from the other three and our solution for this version required a more careful plan for the movement of the pixels.

The remainder of the paper is organized as follows. After preliminaries in Section 2, we give proofs for $(4,8),(8,4),(8,8)$ and $(4,4)$-IP-equivalence in Section 3, Section 4, Section 5 and Section 6, respectively. We conclude in Section 7.

\section{Preliminaries}

For a pixel $p=(x, y)$, we use the notation $\mathrm{N}(p)$ (respectively, $\mathrm{E}(p), \mathrm{s}(p), \mathrm{w}(p))$ to denote the pixel $(x, y+1)$ (respectively, $(x+1, y),(x, y-1),(x-1, y))$. We allow concatenation of these modifiers so that, for example $\mathrm{NE}(p)=\mathrm{N}(\mathrm{E}(p)), \operatorname{NNE}(p)=\mathrm{N}(\mathrm{N}(\mathrm{E}(p)))$, and so on. We use the shorthand $\mathrm{N}^{(0)}(p)=p$ and, for $k>0$, $\mathrm{N}^{(k)}(p)=\mathrm{NN}^{(k-1)}(p)$. We also use the regular expression notations $*$ and + so that, for example $\mathrm{N}^{+}(p)=$ $\left\{\mathrm{N}^{(k)}(p): k>0\right\}$ and $N^{*}(p)=\left\{\mathrm{N}^{(k)}(p): k \geq 0\right\}$.

For a graph $G$, let $V(G)$ and $E(G)$ denote the vertex and edge sets of $G$. The subgraph of $G$ induced by a set of vertices $S \subseteq V(G)$ has vertex set $S$ and edge set $\{v w \in E(G): v, w \in S\}$, and is denoted by $G[S]$. A non-empty graph $G$ is called connected if there is a path between any pair of vertices in $G$, otherwise $G$ is disconnected. A maximal connected subgraph of a $G$ is called a component of $G$. A cut vertex of a connected graph $G$ is a vertex $v$ whose removal disconnects $G$, that is $G[V(G) \backslash\{v\}]$ has at least two components. For brevity we will often write $G \backslash v$ instead of $G[V(G) \backslash\{v\}]$. Note that for any image $I$, since $B_{a}(I)$ is finite, each cut vertex in $W_{b}(I)$ splits $W_{b}(I)$ into $k \geq 2$ components $k-1$ of which are finite and one of which is infinite. 
For a graph $G$ and a vertex $v \in V(G)$, let $\mathrm{A}_{G}(v)$ denote the set of all the vertices in $V(G) \backslash v$ that are adjacent to $v$. Furthermore, let $\mathrm{A}_{G}[v]=\mathrm{A}_{G}(v) \cup v$. We start with two simple but useful observations. The first one is a well known graph theoretic fact.

Observation 1. For a graph $G$, a vertex $v \in V(G)$ and any set $S \subseteq V(G) \backslash\{v\}$, if $G\left[\mathrm{~A}_{G}(v) \cup S\right]$ is connected then $v$ in not a cut-vertex of $G$. As a special case, consider two vertices $v, w \in V(G)$, if $\mathrm{A}_{G}(v) \subseteq \mathrm{A}_{G}[w]$, then $v$ in not a cut-vertex of $G$.

Our second observation gives a sufficient condition for an interchange to preserve connectivity.

Observation 2. For a $B_{a}, W_{b}$-connected image $I$, let $p$ be a black pixel that is not a cut vertex in $B_{a}(I)$ and $q a$ white pixel that is not a cut vertex in $W_{b}(I)$. If $p$ has a white b-neighbour in I other than $q$ and $q$ has a black $a$-neighbour in $I$ other than $p$, then the interchange $\langle p, q\rangle$ preserves $B_{a}, W_{b}$-connectivity.

For a pixel $p=(x, y)$, we say $x$ is the $\mathrm{x}$-coordinate of $p$ and $y$ is the $\mathrm{y}$-coordinate of $p . \mathrm{A} \mathrm{B}_{a}, \mathrm{~W}_{b^{-}}$ connected image $I$ is vertical if all black pixels in $I$ have the same x-coordinate, otherwise $I$ is non-vertical. We prove that each $\mathrm{B}_{a}, \mathrm{~W}_{b}$-connected image $I,(a, b) \in\{(4,8),(8,4),(8,8)\}$ is $(a, b)$-IP-equivalent to some vertical image. Our approach to, or more precisely, the sequence of interchanges used in solving all but the $(4,4)$ version of the problem have some commonalities. We describe these commonalities in the reminder of this section.

\subsection{Our approach to solving $(4,8),(8,4)$, and $(8,8)$ versions of the problem}

To prove that each $\mathrm{B}_{a}, \mathrm{~W}_{b}$-connected image $I,(a, b) \in\{(4,8),(8,4),(8,8)\}$ is $(a, b)$-IP-equivalent to some vertical image we use the following kinds of interchanges only.

For a $\mathrm{B}_{a}, \mathrm{~W}_{b}$-connected image $I$, and an integer $k \geq 1$, we say that $I$ admits a $k$-vertical interchange if there exists a sequence of at most $k$ 8-local interchanges $\left(\left\langle p_{i}, q_{i}\right\rangle: 1 \leq i \leq k\right)$ such that $p_{i}$ is black and it is not a black pixel with minimum $x$-coordinate in $I, q_{i}$ is white, and

- if $k>1$, then for all $i<k, q_{i}=\mathrm{E}\left(p_{i}\right)$ and $q_{k} \in\left\{\mathrm{NW}\left(p_{k}\right), \mathrm{N}\left(p_{k}\right), \mathrm{NE}\left(p_{k}\right)\right\}$

- otherwise, $k=1$, and $q_{1} \in\left\{\mathrm{W}\left(p_{1}\right), \mathrm{NW}\left(p_{1}\right), \mathrm{N}\left(p_{1}\right), \mathrm{NE}\left(p_{1}\right)\right\}$.

Moreover, after each interchange $\left\langle p_{i}, q_{i}\right\rangle$, the resulting image $I^{i}$ is $\mathrm{B}_{a}, \mathrm{~W}_{b}$-connected.

To simplify the exposition in Section 3, Section 4 and Section 5, we will use the term interchange in place of 1-vertical interchange. This will not cause confusion since the only type of interchanges we use in these three section are $k$-vertical interchanges.

Lemma 1. Suppose that each non-vertical $B_{a}, W_{b}$-connected binary image admits a $k$-vertical interchange, for some integer $k \geq 1$. Then every $B_{a}, W_{b}$-connected binary image $I$ is $(a, b)$-IP-equivalent to some vertical image. Furthermore, I can be converted into a vertical image by a sequence of $O\left(k n^{2}\right)$ 8-local interchanges, where $n$ is the number of black pixels in $I$.

Proof. Without loss of generality, assume that minimum x-coordinate of all black pixels of $I$ is 0 and that of all black pixels with $x$-coordinate 0 , the minimum $y$-coordinate is 0 . Let $p_{0}$ be the black pixel $(0,0)$. Define the potential of a black pixel $p=(x, y)$ as $\Phi(p)=x+(k+1)(n-y)$ and define the potential $\Phi(I)$ of image $I$ as the sum the potentials of all black pixels in $I$. Because $p_{0}$ is black and $B_{a}(I)$ is connected, it is easily verified that $\Phi(p)<(2 k+3) n$ for any black pixel $p$ in $I$ and therefore $\Phi(I)<(2 k+3) n^{2}$. Furthermore, any image that has no black pixel with negative $x$-coordinate has non-negative potential. 
It is simple to verify that applying a $k$-vertical interchange to $I$, results in an image $I^{\prime}$ with smaller potential than $I$, that is $\Phi\left(I^{\prime}\right)<\Phi(I)$. Thus by applying at most $(2 k+3) n^{2} k$-vertical interchanges to $I$ we obtain an image $J$ such that $\Phi(J)<0$. However, that cannot occur unless we, at some point, performed an interchange involving a black pixel with $\mathrm{x}$-coordinate 0 which is not possible given the definition of $k$-vertical interchange. We conclude that at some point during the first $(2 k+3) n^{2}$ interchanges we obtained a vertical image.

\section{Maintaining $\mathrm{B}_{4}, \mathrm{~W}_{8}$-Connectivity}

The following lemma is the main step in the proof that two $\mathrm{B}_{4}, \mathrm{~W}_{8}$-connected images $I$ and $J$ differ by a sequence of 8-local interchanges.

Lemma 2. Any non-vertical $\mathrm{B}_{4}, \mathrm{~W}_{8}$-connected binary image I admits a 2-vertical interchange.

Proof. Let $p=(x, y)$ be the pixel such that

1. $p$ is black,

2. $S(p)$ is white,

3. There exists an integer $k \geq 0$ such that all pixels $\mathrm{N}^{(1)}(p), \ldots, \mathrm{N}^{(k)}(p)$ are black and all pixels in $\mathrm{N}^{+} \mathrm{N}^{(k)}(p)$ are white,

4. all pixels in $\mathrm{E}^{+} \mathrm{N}^{*}(p)$ are white, and

5. $y$ is maximum.

Such a pixel always exists because a pixel satisfying the first four conditions can be found in the set of black pixels with maximum $x$-coordinate and a pixel satisfying the fifth condition is guaranteed by finiteness. Furthermore, $p$ is not a pixel with minimum x-coordinate in $I$, as otherwise $I$ would be vertical or $B_{4}(I)$ would be disconnected. We will show that each pixel $p_{i}$ in the 2 -vertical interchange $\left\langle p_{i}, q_{i}\right\rangle, 1 \leq i \leq 2$, is located near $p$. To simplify the exposition, in what follows we will argue that $p_{i}$ is not a black pixel with minimum $x$-coordinate only when it is not obvious. Furthermore, only in the last case, $2 \mathrm{~b}$, will we be using 2 -vertical interchanges. On all other occasions we will be using a 1-vertical interchange, i.e., an interchange $\left\langle p_{1}, q_{1}\right\rangle$ where $q \in\left\{\mathrm{W}\left(p_{1}\right), \mathrm{NW}\left(p_{1}\right), \mathrm{N}\left(p_{1}\right), \mathrm{NE}\left(p_{1}\right)\right\}$. To prove the lemma we distinguish between two main cases.

Case 1: $p$ is not a cut vertex of $B_{4}(I)$. In this case, if $\mathrm{N}(p)$ is black (Figure 2.a) then we can perform the interchange $\langle p, \operatorname{NE}(p)\rangle$. Since $p$ is not a cut vertex of $B_{4}(I)$ and $\operatorname{NE}(p)$ is not a cut vertex of $W_{8}(I)$, Observation 2, implies that this interchange preserves connectivity.

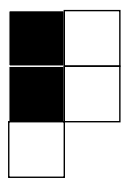

(a)



(b)

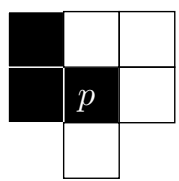

(c)



(d)

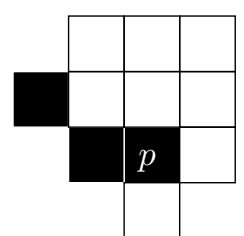

(e)

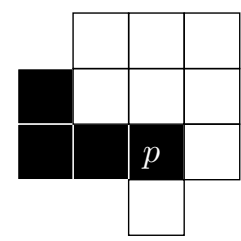

(f)

Figure 2: Illustrating Case 1 in the proof of Lemma 2.

Therefore, we may assume that $\mathrm{N}(p)$ is white. But in this case, $\mathrm{w}(p)$ must be black (Figure 2.b), otherwise $p$ would be an isolated vertex in $B_{4}(I)$. 
If $\mathrm{NW}(p)$ is black (Figure 2.c), then we can perform the interchange $\langle p, \mathrm{~N}(p)\rangle$. Again, neither $p$ nor $\mathrm{N}(p)$ are cut-vertices in their respective graphs, so this interchange preserves connectivity by Observation 2.

Otherwise $\operatorname{NW}(p)$ is white (Figure 2.d). and we claim that the interchange $\langle p, \operatorname{NW}(p)\rangle$ preserves connectivity. Observe that by the choice of $p$, all pixels in $\mathrm{N}^{+}(p)$ and $\mathrm{N}^{+} \mathrm{W}(p)$ are white (Figure 2.d). If $\mathrm{NW}(p)$ is not a cut vertex in $W_{8}(I)$ then the claim follows by Observation 2. The only way in which $\mathrm{NW}(p)$ could be a cut vertex in $W_{8}(I)$ is if $\operatorname{WNW}(p)$ is black (Figure 2.e). But in this case, the choice of $p$ ensures that $\operatorname{WW}(p)$ is black (Figure 2.f) contradicting the assumption that $\mathrm{NW}(p)$ is a cut vertex of $W_{8}(I)$.

Case 2: $p$ is a cut vertex of $B_{4}(I)$. In this case, $\mathrm{N}(p)$ and $\mathrm{w}(p)$ must be black or else $p$ would have less than 2 neighbours in $B_{4}(I)$ and could not be a cut vertex. Also, NW $(p)$ must be white (Figure 3.a) otherwise the graph induced by $\mathrm{A}_{B_{4}}(p)$ would be connected and thus $p$ would not be a cut vertex. By the same reasoning, if the interchange $\langle p, \mathrm{NW}(p)\rangle$ does not preserve connectivity, it is because $\mathrm{NW}(p)$ is a cut vertex in $W_{8}(I)$. We now consider the possible ways in which this can happen.

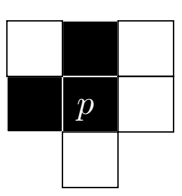

(a)



(b)

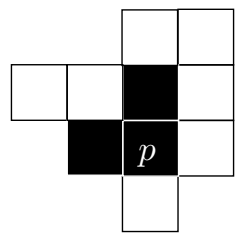

(c)

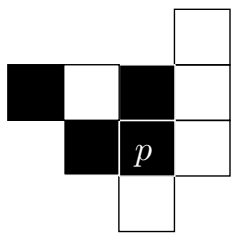

(d)



(e)

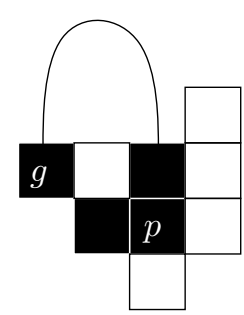

(f)

Figure 3: Illustrating Case 2 in the proof of Lemma 2.

If $\operatorname{NWW}(p)$ is white, (Figure 3.b) then $\mathrm{NN}(p)$ is white (Figure 3.c), otherwise $\mathrm{A}_{W_{8}}(\mathrm{NW}(p)) \subseteq \mathrm{A}_{W_{8}}[\mathrm{NWW}(p)]$ and thus by Observation $1, \mathrm{NW}(p)$ would not be a cut vertex in $W_{8}(I)$. Having $\mathrm{NN}(p)$ white implies by the choice of $p$ that $\mathrm{NN}^{+}(p), \mathrm{WN}^{+}(p)$ and $\mathrm{WWN}^{+}(p)$ are white. In that case the graph induced by $\mathrm{A}_{W_{8}}(\mathrm{NW}(p))$ in $W_{8}(I)$ is connected and again by Observation $1, \mathrm{NW}(p)$ is not a cut vertex in $W_{8}(I)$.

Therefore, assume $\operatorname{NWW}(p)$ is black, (Figure 3.d). Let $g=\mathrm{NWW}(p)$. It is either the case that every path from $g$ to $p$ in $B_{4}(I)$ goes through $\mathrm{w}(p)$ (Figure 3.e) or every path from $g$ to $p$ in $B_{4}(I)$ goes through $\mathrm{N}(p)$ (Figure 3.f). Otherwise, if there is a path through $\mathrm{w}(p)$ and a path through $\mathrm{N}(p)$, then $W_{8}(I)$ would be disconnected; or, if there is neither a path through $\mathrm{w}(p)$ nor $\mathrm{N}(p)$, then $B_{4}(I)$ would be disconnected. Based on that we now have two cases to consider.

Case 2a: Every path from $g$ to $p$ in $B_{4}(I)$ goes through $\mathrm{w}(p)$ (Figure 4.a).

If $\mathrm{WW}(p)$ is black (Figure 4.b), then $\operatorname{NNWW}(p)$ is white, $\operatorname{NNW}(p)$ is black and $\mathrm{NN}(p)$ is white (Figure 4.c), as otherwise $\mathrm{NW}(p)$ is not a cut vertex of $W_{8}(I)$, by Observation 1. However this is not possible due to the choice of $p$.

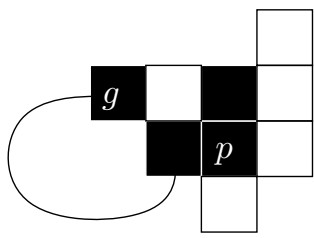

(a)



(b)



(c)

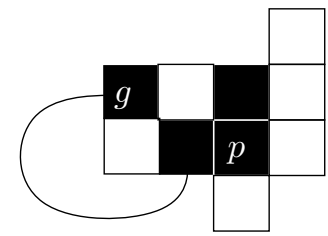

(d)

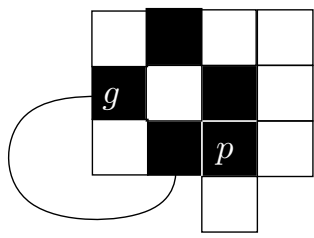

(e)

Figure 4: Illustrating Case 2a in the proof of Lemma 2.

Therefore, $\mathrm{wW}(p)$ is white (Figure 4.d). In this case, we claim that the interchange $\langle\mathrm{w}(p), \mathrm{NW}(p)\rangle$ 
preserves connectivity of the resulting image $I^{1}$.

To see that $W_{8}\left(I^{1}\right)$ is connected, first observe that $W_{8}(I) \backslash \mathrm{NW}(p)$ has two components. (It cannot have three components as otherwise, $\operatorname{NNWW}(p)$ is white, $\operatorname{NNW}(p)$ is black and $\mathrm{NN}(p)$ is white (Figure 4.d), which is impossible due the the choice of $p$.) The finite component of $W_{8}(I) \backslash \mathrm{NW}(p)$ contains $\mathrm{WW}(p)$, and the infinite component contains $\mathrm{s}(p)$. Therefore, to see that $W_{8}\left(I^{1}\right)$ is connected it is enough to observe that $W_{8}\left(I^{1}\right)$ can be obtained by adding $\mathrm{W}(p)$ to $W_{8}(I) \backslash \mathrm{NW}(p)$ where $\mathrm{w}(p)$ is adjacent in $W_{8}\left(I^{1}\right)$ to at least one vertex of the finite component, in particular $\mathrm{wW}(p)$, and at least one vertex of the infinite component, in particular $\mathrm{s}(p)$. To see that $B_{4}\left(I^{1}\right)$ is connected observe that because $\mathrm{w}(p)$ has only two neighbours in $B_{4}(I)$ (namely, $p$ and $\mathrm{sw}(p)$ ), $B_{4}(I) \backslash \mathrm{w}(p)$ has two components, one containing $p$ (and $\mathrm{N}(p)$ ) and the other containing $\operatorname{sw}(p)$ and $g$. Therefore, to see that $B_{4}\left(I^{1}\right)$ is connected it is enough to observe that $B_{4}\left(I^{1}\right)$ can be obtained by adding $\mathrm{NW}(p)$ to $B_{4}(I) \backslash \mathrm{W}(p)$ where $\mathrm{NW}(p)$ is adjacent in $B_{4}\left(I^{1}\right)$ to at least one vertex of the first component, in particular $\mathrm{N}(p)$, and at least one vertex of the second component, in particular $g$.

Case 2b: Every path from $g$ to $p$ in $B_{4}(I)$ goes through $\mathrm{N}(p)$.

Then $\operatorname{ww}(p)$ cannot be black (Figure 5.a), otherwise there is a path from $g$ to $p$ that does not go through $\mathrm{N}(p)$. Having $\mathrm{NW}(p)$ white, and having every path from $g$ to $p$ go through $\mathrm{N}(p)$ implies that $\mathrm{NN}(p)$ is black (Figure 5.b). That further implies at least one of $\{\operatorname{NNW}(p), \operatorname{NNWW}(p)\}$ is white, as otherwise $\operatorname{NW}(p)$ would not be a cut vertex of $W_{8}(I)$ by Observation 1 .

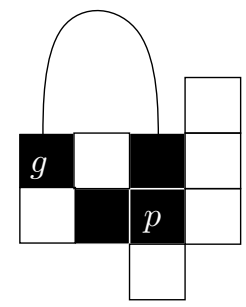

(a)

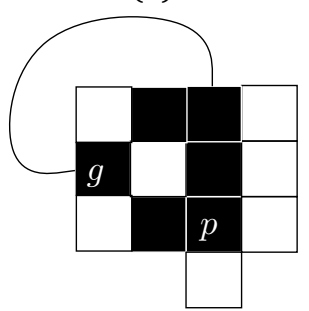

(d)

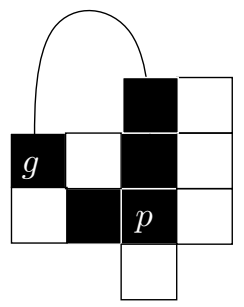

(b)

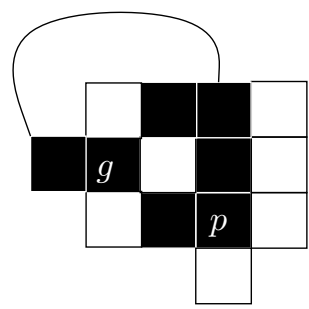

(e)

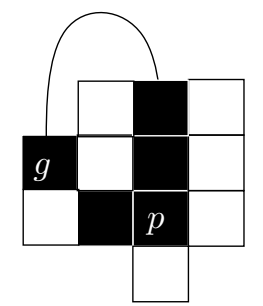

(c)

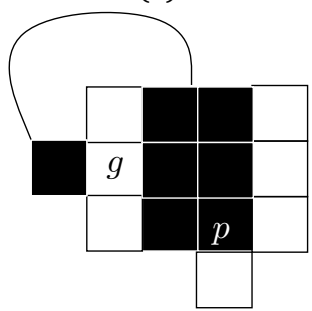

(f)

Figure 5: Illustrating Case $2 \mathrm{~b}$ in the proof of Lemma 2.

First consider the case that $\mathrm{NNW}(p)$ is white (Figure 5.c). We claim that the interchange $\langle\mathrm{N}(p), \mathrm{NW}(p)\rangle$ preserves connectivity of the resulting image $I^{1}$. To see that $W_{8}\left(I^{1}\right)$ is connected, first observe that $W_{8}(I) \backslash$ $\mathrm{NW}(p)$ has two components, the finite of which contains $\mathrm{NNW}(p)$ and infinite of which contains $\mathrm{E}(p)$. Thus $W_{8}\left(I^{1}\right)$ is connected as it can be obtained by adding $\mathrm{N}(p)$ to $W_{8}(I) \backslash \mathrm{NW}(p)$ where $\mathrm{N}(p)$ is adjacent in $W_{8}\left(I^{1}\right)$ to at least one vertex of the finite component, in particular $\operatorname{NNW}(p)$, and at least one vertex of the infinite component, in particular $\mathrm{E}(p)$. To see that $B_{4}\left(I^{1}\right)$ is connected observe that because $\mathrm{N}(p)$ has only two neighbours in $B_{4}(I), B_{4}(I) \backslash \mathrm{N}(p)$ has two components, one containing $p$ (and $\mathrm{w}(p)$ ) and the other containing $g$. Therefore, $B_{4}\left(I^{1}\right)$ is connected as it can be obtained by adding $\mathrm{NW}(p)$ to $B_{4}(I) \backslash \mathrm{N}(p)$ where $\mathrm{NW}(p)$ is adjacent in $B_{4}\left(I^{1}\right)$ to at least one vertex of the first component, in particular $\mathrm{w}(p)$, and at least one vertex of the second component, in particular $g$.

Now consider the case that $\operatorname{NNW}(p)$ is black. Then $\operatorname{NNWW}(p)$ is white (Figure 5.d) and thus $g$ has only one neighbour in $B_{4}(I)$, namely $\mathrm{w}(g)$. Thus $g$ is not a cut vertex in $B_{4}(I)$ and it is not a black pixel with minimum $x$-coordinate in $I$. We claim that the 2 -vertical interchange $\langle g, \mathrm{E}(g)\rangle,\langle\mathrm{N}(p), \operatorname{NNE}(p)\rangle$ preserves connectivity of both resulting images $I^{1}$ and $I^{2} . B_{4}\left(I^{1}\right)$ is connected since $g$ is not a cut vertex in $B_{4}(I)$ and 
since $\mathrm{E}(g)$ has a black pixel in its 4-neighbourhood distinct from $g$ (recall Observation 2). To see that $W_{8}\left(I^{1}\right)$ is connected, first observe that $W_{8}(I) \backslash \mathrm{E}(g)$ has two components, the finite of which contains $\mathrm{N}(g)$ and infinite of which contains $\mathrm{S}(g)$. Thus $W_{8}\left(I^{1}\right)$ is connected as it can be obtained by adding $g$ to $W_{8}(I) \backslash \mathrm{E}(g)$ where $g$ is adjacent in $W_{8}\left(I^{1}\right)$ to at least one vertex of the finite component, in particular $\mathrm{N}(g)$, and at least one vertex of the infinite component, in particular $s(g)$. See Figure 5.f for the resulting image $I^{1}$. It is now simple to verify that the second interchange, $\langle\mathrm{N}(p), \operatorname{NNE}(p)\rangle$ preserves the connectivity of $I^{2}$.

By applying Lemma 1 to convert any binary image $I$ into a vertical image and then converting that image into any other binary image $J$ we obtain our first theorem.

Theorem 1. Any two $\mathrm{B}_{4}, \mathrm{~W}_{8}$-connected images $I$ and $J$, each having $n$ black pixels, are $(4,8)$-IP-equivalent and $I$ can be converted into $J$ using a sequence of $O\left(n^{2}\right)$ 8-local interchanges.

\section{Maintaining $\mathrm{B}_{8}, \mathrm{~W}_{4}$-Connectivity}

Lemma 3. Any non-vertical $\mathrm{B}_{8}, \mathrm{~W}_{4}$-connected binary image I admits a 4-vertical interchange.

Proof. Let $p=(x, y)$ be the pixel such that

1. $p$ is black,

2. $S(p)$ is white,

3. There exists an integer $k \geq 0$ such that all pixels $\mathrm{N}^{(1)}(p), \ldots, \mathrm{N}^{(k)}(p)$ are black and all pixels in $\mathrm{N}^{+} \mathrm{N}^{(k)}(p)$ are white,

4. all pixels in $\mathrm{SE}^{+} \mathrm{N}^{*}(p)$ are white, and

5. $y$ is maximum.

Such a pixel always exists because a pixel satisfying the first four conditions can be found in the set of black pixels with maximum $\mathrm{x}$-coordinate and a pixel satisfying the fifth condition is guaranteed by finiteness. Furthermore, $p$ is not a vertex with minimum x-coordinate in $I$, as otherwise $I$ would be vertical or $B_{8}(I)$ would be disconnected. We will show that each pixel $p_{i}$ in the 4 -vertical interchange $\left\langle p_{i}, q_{i}\right\rangle: 1 \leq i \leq 4$, is located relatively close to $p$. To simplify the exposition, in what follows we will argue that $p_{i}$ is not a black pixel with minimum $x$-coordinate only when it is not obvious. Furthermore, only in the last case, the case $2 \mathrm{~b}$, we will be using $k$-vertical interchanges where $k>1$. On all the other occasions we will be using a 1-vertical interchange, that is an interchange $\left\langle p_{1}, q_{1}\right\rangle$ where $q \in\left\{\mathrm{W}\left(p_{1}\right), \mathrm{NW}\left(p_{1}\right), \mathrm{N}\left(p_{1}\right), \mathrm{NE}\left(p_{1}\right)\right\}$. To prove the lemma we distinguish between two main cases.

Case 1: $p$ is not a cut vertex of $B_{8}(I)$. In this case, if $\mathrm{N}(p)$ is black (Figure 6.a) then we can perform the interchange $\langle p, \operatorname{NE}(p)\rangle$. Since $p$ is not a cut vertex of $B_{8}(I)$ and $\mathrm{NE}(p)$ is not a cut vertex of $W_{4}(I)$, Observation 2 implies that this interchange preserves connectivity.

Therefore, we may assume that $\mathrm{N}(p)$ is white. Then if at least one of $\{\mathrm{NW}(p), \mathrm{W}(p)\}$ is black (Figure 6.b and c), the interchange $\langle p, \mathrm{~N}(p)\rangle$ preserves connectivity since $\mathrm{N}(p)$ is not a cut vertex of $W_{4}(I)$ by the choice of $p$ and Observation 1. Thus assume both $\mathrm{w}(p)$ and $\mathrm{NW}(p)$ are white and $\operatorname{sw}(p)$ is black (Figure 6.d). Then by the choice of $p, \mathrm{wN}^{+}(p)$ is all white. If $\mathrm{w}(p)$ is not a cut vertex of $W_{4}(I)$ then the interchange $\langle p, \mathrm{~W}(p)\rangle$ preserves connectivity. Otherwise, $\mathrm{w}(p)$ is a cut vertex and thus $\mathrm{wW}(p)$ is white and $\operatorname{NWW}(p)$ is black (Figure 6.e). We claim that the interchange $\langle p, \operatorname{NW}(p)\rangle$ preserves connectivity. $W_{4}\left(I^{1}\right)$ can be disconnected only if $\mathrm{NW}(p)$ is a cut vertex in $W_{4}(I)$. In that case $W_{4}(I) \backslash \mathrm{NW}(p)$ has two components, the 
finite of which contains $\mathrm{W}(p)$ and infinite of which contains $\mathrm{E}(p)$. Therefore, to see that $W_{4}\left(I^{1}\right)$ is connected it is enough to observe that $W_{4}\left(I^{1}\right)$ can be obtained by adding $p$ to $W_{4}(I) \backslash \mathrm{NW}(p)$ where $p$ is adjacent in $W_{4}\left(I^{1}\right)$ to at least one vertex of the finite component, in particular $\mathrm{w}(p)$, and at least one vertex of the infinite component, in particular $\mathrm{E}(p)$.



(a)

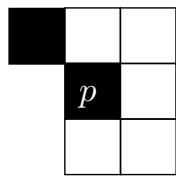

(b)

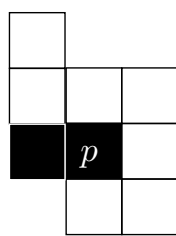

(c)



(d)



(e)

Figure 6: Illustrating Case 1 in the proof of Lemma 3.

Case 2: $p$ is a cut vertex of $B_{8}(I)$. In this case $\mathrm{w}(p)$ is white, otherwise $\mathrm{A}_{B_{8}}(p) \subseteq \mathrm{A}_{B_{8}}[\mathrm{w}(p)]$ and by Observation $1, p$ would not be a cut vertex in $B_{8}(I)$. Similarly, $\mathrm{sw}(p)$ has to be black (Figure 7.a) as otherwise, the graph induced by $\mathrm{A}_{B_{8}}(p)$ would be connected and $p$ would not be a cut vertex in $B_{8}(I)$. If $\mathrm{W}(p)$ is not a cut vertex of $W_{4}(I)$ then the interchange $\langle p, \mathrm{~W}(p)\rangle$ preserves connectivity. Therefore, assume $\mathrm{W}(p)$ is a cut vertex of $W_{4}(I)$. Then $\mathrm{WW}(p)$ and $\mathrm{NW}(p)$ must be white and $\mathrm{NWW}(p)$ must be black (Figure 7.b), as otherwise the graph induced by $\mathrm{A}_{W_{4}}(\mathrm{w}(p))$ would be connected and $\mathrm{w}(p)$ would not be a cut vertex in $W_{4}(I)$. All together this implies that $\mathrm{N}(p)$ is black as otherwise $p$ is not a cut vertex of $B_{8}(I)$ (Figure 7.c). Let $g=\mathrm{NWW}(p)$.

It is either the case that every path from $g$ to $p$ in $B_{8}(I)$ goes through $\operatorname{sw}(p)$ (Figure 7.d) or every path from $g$ to $p$ in $B_{8}(I)$ goes through $\mathrm{N}(p)$ (Figure 7.e). Otherwise, if there is a path through $\mathrm{sw}(p)$ and a path through $\mathrm{N}(p)$, then $W_{4}(I)$ would be disconnected; or, if there is neither a path through $\mathrm{sw}(p) \operatorname{nor} \mathrm{N}(p)$, then $B_{8}(I)$ would be disconnected.

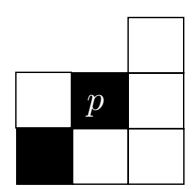

(a)

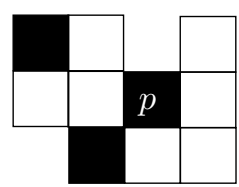

(b)

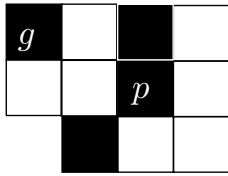

(c)

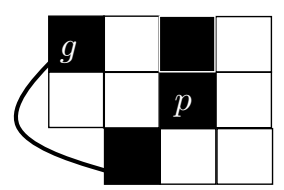

(d)

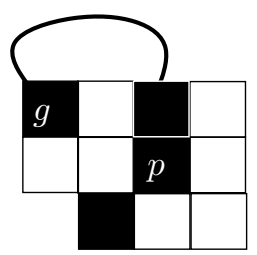

(e)

Figure 7: Illustrating Case 2 in the proof of Lemma 3.

Case 2a: Every path from $g$ to $p$ in $B_{8}(I)$ goes through $\mathrm{sw}(p)$. In that case we claim that the interchange $\langle p, \mathrm{NW}(p)\rangle$ preserves connectivity. To see that $B_{8}\left(I^{1}\right)$ is connected first observe that $B_{8}(I) \backslash p$ has two components, one containing $\mathrm{sw}(p)$ and $g$ and the other containing $\mathrm{N}(p)$. Therefore, $B_{8}\left(I^{1}\right)$ is connected since it can be obtained by adding $\mathrm{NW}(p)$ to $B_{8}(I) \backslash p$ where $\mathrm{NW}(p)$ is adjacent in $B_{8}\left(I^{1}\right)$ to at least one vertex of the first component, in particular $g$, and at least one vertex of the second component, in particular $\mathrm{N}(p)$. To see that $W_{4}\left(I^{1}\right)$ is connected first observe that $W_{4}(I) \backslash \mathrm{NW}(p)$ has two components, the finite of which contains $\mathrm{W}(p)$ and infinite of which contains $\mathrm{s}(p)$. Therefore, $W_{4}\left(I^{1}\right)$ is connected since it can be obtained by adding $p$ to $W_{4}(I) \backslash \mathrm{NW}(p)$ where $p$ is adjacent in $W_{4}\left(I^{1}\right)$ to at least one vertex of the finite component, in particular $\mathrm{W}(p)$, and at least one vertex of the infinite component, in particular $\mathrm{s}(p)$.

Case 2b: Every path from $g$ to $p$ in $B_{8}(I)$ goes through $\mathrm{N}(p)$. Use Figure 8.a as reference throughout this proof.

Let $l$ be the black pixel with minimum y-coordinate in $\mathrm{wNN}^{+}(p)$. Such a pixel has to exist as otherwise there would be no path from $g$ to $p$. For the same reason, all the pixels in $\left\{\mathrm{ES}^{+}(l) \cap \mathrm{N}^{*}(p)\right\}$ are black. By the choice of $l$, all the pixels in $\mathrm{s}^{+}(l) \cap \mathrm{WN}^{*}(p)$ are white (Figure 8.b). 
If $\mathrm{s}(l)$ is not a cut vertex in $W_{4}(I)$ then the interchange $\langle\mathrm{SE}(l), \mathrm{s}(l)\rangle$ preserves connectivity. Thus assume $\mathrm{s}(l)$ is a cut vertex in $W_{4}(I)$. Then $\mathrm{s}(l)$ has to have at least two neighbours in $W_{4}(I)$ and thus $\operatorname{ss}(l)$ is white and $\operatorname{sw}(l)$ is white. Furthermore, for $\mathrm{s}(l)$ to be a cut vertex $\operatorname{ssw}(l)$ has to be black. Since $\operatorname{ssw}(l) \in \mathrm{N}^{*}(g), \operatorname{ssse}(l)$ is black and $\operatorname{sss}(l)$ is white (Figure 8.c).

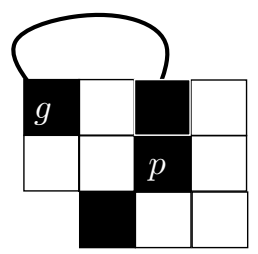

(a)

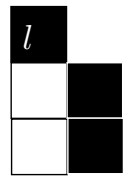

(b)

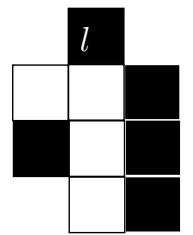

(c)

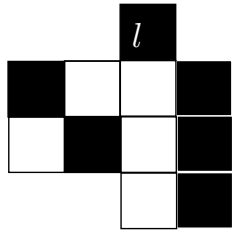

(d)

Figure 8: Illustrating Case $2 \mathrm{~b}$ in the proof of Lemma 3.

Note that having $\operatorname{sw}(l)$ white implies that there is a black pixel in $w_{w N}^{*} s^{*}(l)$ as otherwise, there would be no path from $g$ to $p$ in $B_{8}(I)$. Therefore, $\operatorname{ssw}(l)$ is not a black pixel with minimum $x$-coordinate. If $\operatorname{ssw}(l)$ is not a cut vertex in $B_{8}(I)$ then clearly the interchange $\langle\operatorname{ssw}(l), \mathrm{s}(l)\rangle$ is valid since it preserves connectivity and since $\operatorname{ssw}(l)$ is not a black pixel with minimum $x$-coordinate. Thus assume $\operatorname{ssw}(l)$ is a cut vertex in $B_{8}(I)$. Then ssww $(l)$ cannot be black, as otherwise $A_{B_{8}}(\operatorname{ssw}(l)) \subseteq \mathrm{A}_{B_{8}}[\operatorname{ssww}(l)]$ and thus $\operatorname{ssw}(l)$ would not be a cut vertex. Similarly, $\operatorname{sww}(l)$ is black (Figure 8.d).

Let $h$ be the black pixel with minimum y-coordinate in $\mathrm{N}^{*}(g)$ such that $\mathrm{N}(h)$ is white. Let $B$ denote the set of all black pixels in-between, and including, $g$ and $h$. That is, $B$ is the set of black pixels in $\left\{\mathrm{N}^{*}(g) \cap \mathrm{s}^{*}(h)\right\}$. For the reminder of the proof refer to Figures 8.a and 8.d as reference for the position of $h$. To complete the proof we distinguish between two cases:

Case 2b-I: There exist a black pixel $z \in B$ such that $\mathrm{W}(z)$ is black. Let $z$ be such a pixel with minimum $\mathrm{y}$-coordinate. Since $\mathrm{A}_{B_{8}}(z) \subseteq \mathrm{A}_{B_{8}}[\mathrm{~W}(z)], z$ is not a cut vertex in $B_{8}(I)$. Therefore, if both $\mathrm{N}(z)$ and $\mathrm{NN}(z)$ are white, then the interchange $\langle z, \operatorname{NE}(z)\rangle$ preserves connectivity (Figure 9.a). Thus assume that at least one of $\{\mathrm{N}(z), \mathrm{NN}(z)\}$ is black.



(a)

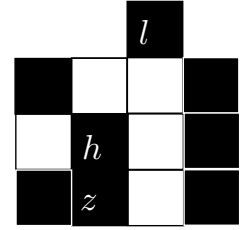

(b)



(c)

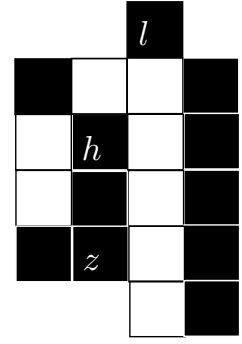

(d)

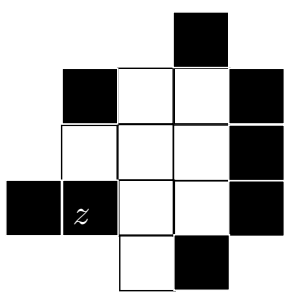

(e)

Figure 9: Illustrating Case 2b-I in the proof of Lemma 3.

Consider the position of $z \in B$. Firstly, $z \neq \operatorname{ssw}(l)$ since $z$ is not a cut vertex of $B_{8}(I)$ and by our assumption $\operatorname{ssw}(l)$ is. If $z=\operatorname{sssw}(l)$ (in which case $h=\mathrm{N}(z)$ ) then $\mathrm{w}(h)$ is not cut vertex in $W_{4}(I)$ and the interchange $\langle h, \mathrm{~W}(h)\rangle$ preserves connectivity (Figure 9.b). Therefore, $z \in \mathrm{wSSss}^{+}(l) \cap \mathrm{N}^{*}(g)$.

To resolve this case we will use the following simple observation. For any set of consecutive black pixels $\left\{v_{1}, \ldots v_{t}\right\}$ in $\mathrm{N}^{+}(p)$ such that each $\mathrm{E}\left(v_{i}\right), 1<=i<=t$, is white and $\mathrm{N}\left(v_{t}\right)$ is black, the sequence of interchanges $\left(\left\langle v_{i}, \mathrm{E}\left(v_{i}\right)\right\rangle\right), 1<=i<=t$ preserve connectivity of each of the resulting images in the sequence (see the final image in Figure 9.c). We call such a set of pixels, $t$-block at $v_{1}$.

By the position of $z$ with respect to $l$, it follows that there is a 3-block at $\operatorname{EE}(z)$ (in the worst case $z=\operatorname{ssssw}(l)$ (as an example consider Figure 9.d). Perform a 4 -vertical interchange $(\langle\operatorname{EE}(z), \operatorname{EEE}(z)\rangle$, 
$\langle\operatorname{NEE}(z), \operatorname{NeEE}(z)\rangle,\langle\operatorname{NNEE}(z), \operatorname{NNEEE}(z)\rangle,\langle z, \operatorname{NE}(z)\rangle)$. Each of the three images $I^{1}, I^{2}$ and $I^{3}$ are clearly $\mathrm{B}_{8}, \mathrm{~W}_{4^{-}}$ connected. $W_{4}\left(I^{4}\right)$ is connected since $W_{4}\left(I^{3}\right) \backslash \mathrm{NE}(z)$ has at most two components one containing $\mathrm{E}(z)$ and the other $\mathrm{N}(z)$ (if $\mathrm{N}(z)$ is white) (as an example consider (Figure 9.e)). In any case, $W_{4}\left(I^{4}\right)$ is connected since it can be obtained by adding $z$ to $W_{4}\left(I^{3}\right) \backslash \mathrm{NE}(z)$ where $z$ is adjacent in $W_{4}\left(I^{4}\right)$ to $\mathrm{E}(z)$ and $\mathrm{N}(z)$ (if white). $B_{8}\left(I^{4}\right)$ is connected by Observation 2 since $z$ is not a cut vertex in $B_{8}\left(I^{3}\right)$ and $\mathrm{NE}(z)$ has at least one black pixel in its 8-neighbourhood other than $z$, namely $\mathrm{N}(z)$ or $\mathrm{NN}(z)$ (one of them has to be black by the assumption from the beginning of case $2 \mathrm{~b}-\mathrm{I}$ ).

Case 2b-II: For each pixel $z \in B, \mathrm{~W}(z)$ is white. That implies that at least one of $\{\mathrm{NW}(h), \operatorname{sw}(g)\}$ is black as otherwise there would be no path in $B_{8}(I)$ from $g$ to $p$. Every such path goes through either $\operatorname{sw}(g)$ or $\operatorname{NW}(h)$.

First consider the case that $h=g$ and thus $\mathrm{N}(g)$ is white. If both $\mathrm{NW}(g)$ and $\operatorname{sw}(g)$ are black (Figure 7.a), then the interchange $\langle g, \mathrm{~W}(g)\rangle$ preserves connectivity. Otherwise, if one of $\{\mathrm{NW}(g), \operatorname{sw}(g)\}$ is white (Figure 7.b and c), then the 2-vertical interchange $(\langle g, \mathrm{E}(g)\rangle,\langle\operatorname{EE}(g), \operatorname{EEEN}(g)\rangle)$ preserves the connectivity of both images $I^{1}$ and $I^{2}$.

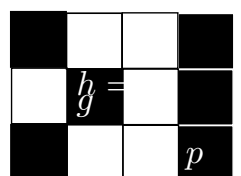

(a)

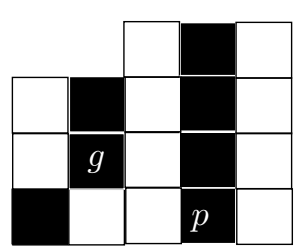

(d)

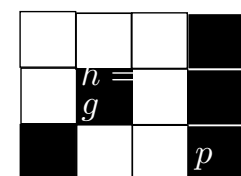

(b)

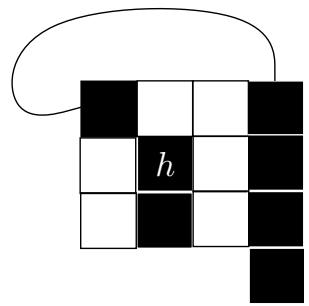

(e)



(c)

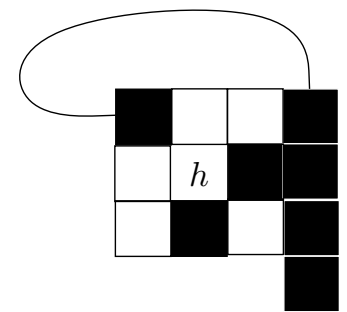

(f)

Figure 10: Illustrating Case 2b-II in the proof of Lemma 3.

Thus assume $h \neq g$ and consider the case the all paths from $g$ to $p$ go through $\operatorname{sw}(g)$. Since $h \neq g$, $\mathrm{N}(g)$ is black (Figure 10.d). We claim the interchange $\langle g, \mathrm{NE}(g)\rangle$ preserves connectivity. To see that $W_{4}\left(I^{1}\right)$ is connected, observe that $W_{4}(I) \backslash \mathrm{NE}(g)$ has two components, the finite one containing $\mathrm{W}(g)$ and the infinite one containing $\mathrm{E}(g)$. Therefore, $W_{4}\left(I^{1}\right)$ is connected since it can be obtained by adding $g$ to $W_{4}(I) \backslash \operatorname{NE}(g)$ where $g$ is adjacent in $W_{4}\left(I^{1}\right)$ to a vertex of the finite component, in particular $\mathrm{w}(g)$, and a vertex of the infinite component, in particular $\mathrm{E}(g)$. To see that $B_{8}\left(I^{1}\right)$ is connected observe that $B_{8}(I) \backslash g$ has two components, one containing $\mathrm{N}(g)$ and the other $\mathrm{sw}(g), p$ and $\mathrm{N}(p)$. Therefore, $B_{8}\left(I^{1}\right)$ is connected since it can be obtained by adding $\mathrm{NE}(g)$ to $B_{8}(I) \backslash g$ where $\mathrm{NE}(g)$ is adjacent in $B_{8}\left(I^{1}\right)$ to a vertex of the first component, in particular $\mathrm{N}(g)$, and a vertex of the second component, in particular $\mathrm{N}(p)$.

Finally, assume $h \neq g$ and all the paths from $g$ to $p$ go through $\mathrm{NW}(h)$. Since $h \neq g, \mathrm{~s}(h)$ is black (Figure 10.e). We claim that the 2-vertical interchange $(\langle h, \mathrm{E}(h)\rangle,\langle\operatorname{EE}(h), \operatorname{EEEN}(h)\rangle)$ preserves connectivity of both images $I^{1}$ and $I^{2}$. To see that $W_{4}\left(I^{1}\right)$ is connected, observe that $W_{4}(I) \backslash \mathrm{E}(h)$ has two components, the finite one containing $\mathrm{N}(h)$ and the infinite one containing $\mathrm{w}(h)$. Therefore, $W_{4}\left(I^{1}\right)$ is connected since it can be obtained by adding $h$ to $W_{4}(I) \backslash \mathrm{E}(h)$ where $h$ is adjacent in $W_{4}\left(I^{1}\right)$ to a vertex of the finite component, in particular $\mathrm{N}(h)$, and a vertex of the infinite component, in particular $\mathrm{w}(h)$. To see that $B_{8}\left(I^{1}\right)$ is connected observe that $B_{8}(I) \backslash h$ has two components, one containing $\mathrm{NW}(h)$ and $p$ (and $\operatorname{EE}(h)$ ) and the other $\mathrm{s}(h$ ). Therefore, $B_{8}\left(I^{1}\right)$ is connected since it can be obtained by adding $\mathrm{E}(h)$ to $B_{8}(I) \backslash h$ where $\mathrm{E}(h)$ is adjacent to a vertex of the first component, in particular $\operatorname{EE}(h)$, and a vertex of the second component, in particular $\mathrm{s}(h)$. See Figure 10.f for the resulting image $I^{1}$. It is now simple to verify that the second interchange, $\langle\operatorname{EE}(h), \operatorname{EEN}(h)\rangle$ preserves the connectivity of $I^{2}$. 


\section{Lemma 1 and Lemma 3 imply the following theorem.}

Theorem 2. Any two $\mathrm{B}_{8}, \mathrm{~W}_{4}$-connected images $I$ and $J$ each having $n$ black pixels are $(8,4)$-IP-equivalent and $I$ can be converted into $J$ using a sequence of $O\left(n^{2}\right)$ 8-local interchanges.

\section{Maintaining $\mathrm{B}_{8}, \mathrm{~W}_{8}$-Connectivity}

Lemma 4. Any non-vertical $\mathrm{B}_{8}, \mathrm{~W}_{8}$-connected binary image I admits a 1-vertical interchange.

Proof. Let $p=(x, y)$ be the pixel defined exactly as in the proof of Lemma 3. We will show that each pixel $p_{1}$ in the 1-vertical interchange $\left\langle p_{1}, q_{1}\right\rangle$, where $q_{1} \in\left\{\mathrm{W}\left(p_{1}\right), \mathrm{NW}\left(p_{1}\right), \mathrm{N}\left(p_{1}\right), \mathrm{NE}\left(p_{1}\right)\right\}$ must exist somewhere near $p$. To prove the lemma we distinguish between two main cases.

Case 1: $p$ is not a cut vertex of $B_{8}(I)$. In this case, if $\mathrm{N}(p)$ is black (Figure 11.a) then we can perform the interchange $\langle p, \mathrm{NE}(p)\rangle$. Since $p$ is not a cut vertex of $B_{8}(I)$ and $\mathrm{NE}(p)$ is not a cut vertex of $W_{8}(I)$, Observation 2, implies that this interchange preserves connectivity.

Therefore, we may assume that $\mathrm{N}(p)$ is white. Then if at least one of $\{\mathrm{NW}(p), \mathrm{W}(p)\}$ is black (Figure 11.b and c), the interchange $\langle p, \mathrm{~N}(p)\rangle$ preserves connectivity since $\mathrm{N}(p)$ is not a cut vertex of $W_{8}(I)$ by the choice of $p$ and Observation 1. Thus assume both $\mathrm{W}(p)$ and $\mathrm{NW}(p)$ are white and $\operatorname{sw}(p)$ is black (Figure 11.d). Then by the choice of $p, \mathrm{WN}^{+}(p)$ is white. That implies that the graph induced by $\mathrm{A}_{W_{8}}(\mathrm{NW}(p))$ is connected and that $\mathrm{NW}(p)$ is not a cut vertex of $W_{8}(I)$. Therefore, if $\mathrm{wW}(p)$ is black (Figure 11.e) the interchange $\langle p, \mathrm{NW}(p)\rangle$ preserves connectivity by Observation 2. Finally, if $\mathrm{ww}(p)$ is white (Figure 11.f), then the graph induced by $\left\{\mathrm{A}_{W_{8}}(\mathrm{~W}(p)) \cup \mathrm{E}(p)\right\}$ is connected thus by Observation $1, \mathrm{~W}(p)$ is not a cut vertex in $W_{8}(I)$, and the interchange $\langle p, \mathrm{~W}(p)\rangle$ preserves connectivity.

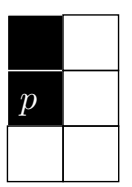

(a)

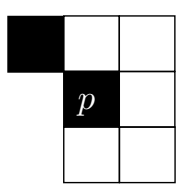

(b)



(c)

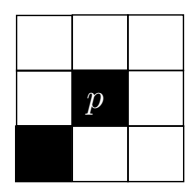

(d)

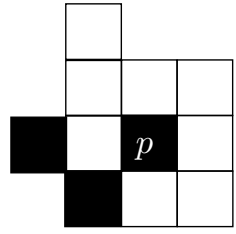

(e)



(f)

Figure 11: Illustrating Case 1 in the proof of Lemma 4.

Case 2: $p$ is a cut vertex of $B_{8}(I)$. In this case $\mathrm{w}(p)$ is white, otherwise $A_{B_{8}}(p) \subseteq \mathrm{A}_{B_{8}}[\mathrm{w}(p)]$ and by Observation 1, $p$ would not be a cut vertex in $B_{8}(I)$. Similarly, $\operatorname{sw}(p)$ has to be black (Figure 12.a) as otherwise, the graph induced by $\mathrm{A}_{B_{8}}(p)$ would be connected and $p$ would not be a cut vertex in $B_{8}(I)$. Finally, for the same reason, at least one of $\{\mathrm{N}(p), \mathrm{NW}(p)\}$ has to be black. Since $p$ is a cut vertex, each path from $\{\mathrm{N}(p), \mathrm{NW}(p)\}$ to $\mathrm{sw}(p)$ goes through $p$.

Assume first that $\mathrm{NW}(p)$ is black. (Figure 12.b). Then $\mathrm{WW}(p)$ has to be white (Figure 12.c) as otherwise $p$ is not cut vertex of $B_{8}(I)$ since the path $\mathrm{NW}(p), \mathrm{WW}(p), \mathrm{sW}(p)$ does not go through $p$. We claim that $\mathrm{W}(p)$ is not a cut vertex of $W_{8}(I)$ and thus that the interchange $\langle p, \mathrm{~W}(p)\rangle$ preserves connectivity. $W_{8}\left(I^{1}\right)$ can only be disconnected if $B_{8}\left(I^{1}\right)$ contains a cycle $C$ in which each pair of consecutive pixels in $C$ are 4-neighbours in $I^{1}$ (that is, $C$ is a cycle in $B_{4}\left(I^{1}\right)$ - recall the observation made on page 1 with regards to the connectivity of images), we call such a cycle a 4-neighbourhood cycle. Thus if $\mathrm{w}(p)$ is a cut vertex of $W_{8}(I)$ then $B_{8}\left(I^{1}\right)$ has a 4-neighbourhood cycle $C$ containing $\mathrm{w}(p)$. Every 4-neighbourhood cycle $C$ containing $\mathrm{W}(p)$ has to contain two vertices from the 4-neighbourhood of $\mathrm{w}(p)$ in $B_{8}\left(I^{1}\right) . \mathrm{w}(p)$ has only two such neighbours in $B_{8}\left(I^{1}\right)$ (Figure 12.c), namely $\mathrm{NW}(p)$ and $\mathrm{sw}(p)$. However having $C$ contain $\mathrm{NW}(p), \operatorname{sw}(p)$ and $\mathrm{w}(p)$ 




(a)

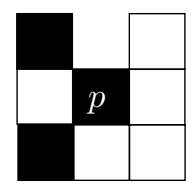

(b)

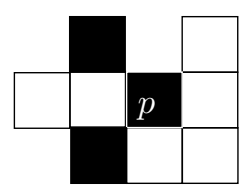

(c)

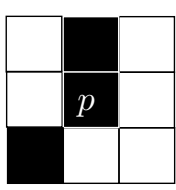

(d)



(e)



(f)

Figure 12: Illustrating Case 2 in the proof of Lemma 4.

implies that there is a path in $B_{8}(I)$ between $\mathrm{NW}(p)$ and $\mathrm{sw}(p)$ that does not go through $p$ which contradicts the assumption that $p$ is a cut vertex in $B_{8}(I)$.

Assume finally that $\mathrm{NW}(p)$ is white. Then as noted above $\mathrm{N}(p)$ is black (Figure 12.d). If $\mathrm{w}(p)$ is not a cut vertex of $W_{8}(I)$ then the interchange $\langle p, \mathrm{~W}(p)\rangle$ preserves connectivity. Thus assume $\mathrm{w}(p)$ to be a cut vertex of $W_{8}(I)$. The only black pixels in the 4-neighborhood of $\mathrm{w}(p)$ in $\left(B_{8}(I) \backslash p\right)$ are $\mathrm{sw}(p)$ and possibly $\mathrm{wW}(p)$. Thus by the same arguments as in the previous paragraph, $\left(B_{8}(I) \backslash p\right) \cup \mathrm{w}(p)$ has a 4neighbourhood cycle $C_{1}$ that has as consecutive vertices $\mathrm{ww}(p), \mathrm{w}(p), \mathrm{sw}(p)$. Therefore, $\mathrm{ww}(p)$ is black and there is a path from $\mathrm{wW}(p)$ to $\operatorname{sw}(p)$ that does not go through $p$ (Figure 12.e). We claim that the interchange $\langle p, \mathrm{NW}(p)\rangle$ preserves connectivity. $B_{8}\left(I^{1}\right)$ is clearly connected. $W_{8}\left(I^{1}\right)$ can only be disconnected if $\mathrm{NW}(p)$ is a cut vertex of $W_{8}(I)$. In that case $\operatorname{NWW}(p)$ has to be black, as otherwise the graph induced by $\mathrm{A}_{W_{8}}(\mathrm{NW}(p)) \cup\{\mathrm{NE}(p), \mathrm{E}(p), \mathrm{S}(p)\}$ would be connected and, by Observation 1, $\mathrm{NW}(p)$ would not be a cut vertex of $W_{8}(I)$. Therefore, since $\operatorname{NWW}(p)$ is black, $\operatorname{NNW}(p)$ has to be white (Figure 12.f) as otherwise, there would be a path from $\operatorname{sw}(p)$ to $\mathrm{N}(p)$ that does not contain $p$ contradicting the assumption that $p$ is a cut vertex. Now the only black pixels in the 4-neighborhood of $\mathrm{NW}(p)$ are $\mathrm{NWW}(p)$ and $\mathrm{N}(p)$. Thus by the same arguments as in the previous paragraph, $\left(B_{8}(I) \backslash p\right) \cup \mathrm{NW}(p)$ has a 4-neighbourhood cycle $C_{2}$ that has as consecutive vertices $\mathrm{N}(p), \mathrm{NW}(p), \mathrm{NWW}(p)$ (Figure 12.f). That however implies again a path in $B_{8}(I)$ from $\mathrm{sw}(p)$ to $\mathrm{N}(p)$ that does not contain $p$, contradicting the assumption that $p$ is a cut vertex.

Lemma 1 and Lemma 4 imply the following theorem.

Theorem 3. Any two $\mathrm{B}_{8}, \mathrm{~W}_{8}$-connected images $I$ and $J$ each having $n$ black pixels are $(8,8)$-IP-equivalent and $I$ can be converted into $J$ using a sequence of $O\left(n^{2}\right)$ 8-local interchanges.

We should note that if $I$ and $J$ are $\mathrm{B}_{4}, \mathrm{~W}_{8}$-connected or $\mathrm{B}_{8}, \mathrm{~W}_{4}$-connected then (8,8)-IP-equivalence of $I$ and $J$ follows from Theorem 1 and Theorem 2 (even if $I$ is $\mathrm{B}_{4}, \mathrm{~W}_{8}$-connected and $J$ is $\mathrm{B}_{8}, \mathrm{~W}_{4}$-connected).

\section{Maintaining $\mathrm{B}_{4}, \mathrm{~W}_{4}$-Connectivity}

Our approach for solving the $\mathrm{B}_{4}, \mathrm{~W}_{4}$ version of the problem is significantly different from that used in the previous three versions. The width of an image $I$, is defined as one plus the difference between the maximum and the minimum $x$-coordinate of the black pixels in $I$. For example, a $\mathrm{B}_{a}, \mathrm{~W}_{b}$-connected image has width one if and only if it is vertical. We will prove that the width of every non-vertical $\mathrm{B}_{4}, \mathrm{~W}_{4}$-connected image $I$ can be reduced by one after $O\left(n^{3}\right)$ interchanges. That will imply the desired result, namely that any two $\mathrm{B}_{4}, \mathrm{~W}_{4}$-connected images $I$ and $J$ each having $n$ black pixels are $(4,4)$-IP-equivalent and that $I$ can be converted into $J$ using a sequence of $O\left(n^{4}\right)$ interchanges.

We will make use of the following notions defined on a $\mathrm{B}_{4}, \mathrm{~W}_{4}$-connected image $I$. A pixel $p$ is an elbow in $I$ if it is black and each pixel in $\{\mathrm{E}(p), \mathrm{s}(p), \mathrm{SE}(p)\}$ is white. If in addition $p$ is a cut vertex in $B_{4}(I)$, we say that $p$ is a cut elbow in $I$. Note that if $p$ is a cut elbow, then $\mathrm{N}(p)$ and $\mathrm{W}(p)$ are black and $\mathrm{NW}(p)$ is 
white. Consider a (possibly empty) set of elbows $\left\{p_{i}: 1 \leq i \leq k, k \geq 0\right\}$ in $I$, such that for each $1 \leq i<k$, $p_{i}=\operatorname{NNWW}\left(p_{i+1}\right)$. We say that $I$ admits a $k$-diagonal interchange at $p_{k}$ if

1. $p_{1}$ is a cut elbow and after each interchange $\left\langle p_{i}, \operatorname{NW}\left(p_{i}\right)\right\rangle$, in the sequence $\left(\left\langle p_{i}, \operatorname{NW}\left(p_{i}\right)\right\rangle: 1 \leq i \leq k\right)$, the resulting image $I^{i}$ is $\mathrm{B}_{4}, \mathrm{~W}_{4}$-connected, or

2. $p_{1}$ is not a cut elbow, and after each interchange $\left\langle p_{i}, \operatorname{SE}\left(p_{i}\right)\right\rangle$, in the sequence $\left(\left\langle p_{i}, \operatorname{SE}\left(p_{i}\right)\right\rangle: 1 \leq i \leq\right.$ $k-1)$, the resulting image $I^{i}$ is $\mathrm{B}_{4}, \mathrm{~W}_{4}$-connected.

Lemma 5. Any $\mathrm{B}_{4}, \mathrm{~W}_{4}$-connected binary image I with an elbow pixel $p$ admits a $k$-diagonal interchange at $p$, for some $k \geq 0$, such that in the final image $J$, $p$ is either an elbow but not a cut elbow in $J$, or $p$ is white. Furthermore, the width of $J$ is at most the width of $I$.

Proof. If $p$ is not a cut elbow in $I$, then the statement is trivial, that is, $I$ admits a $(k=0)$-diagonal interchange. Thus assume $p$ is a cut elbow in $I$. Then $\mathrm{N}(p)$ and $\mathrm{w}(p)$ are both black and $\mathrm{NW}(p)$ is white. If $\mathrm{NW}(p)$ is not a cut vertex in $W_{4}(I)$ then the interchange $\langle p, \mathrm{NW}(p)\rangle$ is a $(k=1)$-diagonal interchange at $p$ of the first type (in this case $p_{1}=p$ ). Thus assume $\mathrm{NW}(p)$ is a cut vertex in $W_{4}(I)$. That implies that $\mathrm{NNWW}(p)$ is an elbow in $I$. Consider the set of all elbows in $\mathrm{N}^{j} \mathrm{~W}^{j}(p)$, for all $j \geq 0$. Let $p_{1}$ be the elbow with the smallest $y$-coordinate in that set such that either

(a) $p_{1}$ is a cut elbow but $\mathrm{NW}\left(p_{1}\right)$ is white and not a cut vertex in $W_{4}(I)$, or

(b) $p_{1}$ is not a cut elbow.

Since the number of black pixels is finite such a pixel $p_{1}$ has to exist. Furthermore, by the above assumption $p_{1} \neq p$. All this implies that $I$ has a set of elbows $p_{1}, \ldots, p_{k}=p, k \geq 2$, where for each $1 \leq i<k$, $p_{i}=\operatorname{NNWW}\left(p_{i+1}\right)$; and, for each $i>1, p_{i}$ is a cut elbow in $I$.

There are two cases to consider depending on whether $p_{1}$ is a cut elbow or not, see (a) and (b) above. If $p_{1}$ is a cut elbow we will show that $I$ admits a $k$-diagonal interchange at $p$ of the first type. Otherwise, $I$ admits a $k$-diagonal interchange at $p$ of the second type. It is simple to observe that in the first case that implies that $p$ is white in the final image $J$, and in the second case $p$ is an elbow but not a cut elbow in $J$. Also in both cases the width of the final image $J$ does not exceed that of $I$.

First consider the case, (a), that $p_{1}$ is a cut elbow in $I$. Then, by the choice of $p_{1}, \operatorname{NW}\left(p_{1}\right)$ is white and not a cut vertex in $W_{4}(I)$. Furthermore, since $p_{1}$ is a cut elbow in $I, \mathrm{~N}\left(p_{1}\right)$ and $\mathrm{w}\left(p_{1}\right)$ are black. Then the interchange $\left\langle p_{1}, \mathrm{NW}\left(p_{1}\right)\right\rangle$ preserves the connectivity of the resulting image $I^{1}$. Moreover, since $\mathrm{W}\left(p_{1}\right)$ is black the width of $I^{1}$ is at most that of $I$. Now, in $I^{1}, \mathrm{NW}\left(p_{2}\right)$ is not a cut vertex (anymore) in $W_{4}\left(I^{1}\right)$ and $p_{2}$ is a cut elbow. Thus in $I_{1}$ the elbow $p_{2}$ plays the role $p_{1}$ played in $I$. Therefore, by an easy induction (on $k$ ) we get that $I$ admits a $k$-diagonal interchange at $p$ of the first type.

Finally, consider the case, (b), that $p_{1}$ is not a cut elbow in $I$. Since $p_{2}$ is a cut elbow in $I, \mathrm{~N}\left(p_{2}\right)$ and $\mathrm{W}\left(p_{2}\right)$ are black and $\mathrm{NW}\left(p_{2}\right)$ (that is, $\left.\mathrm{SE}\left(p_{1}\right)\right)$ is white. We claim that the interchange $\left\langle p_{1}, \mathrm{SE}\left(p_{1}\right)\right\rangle$ preserves the connectivity of the resulting image $I^{1}$. By Observation $2, B_{4}\left(I^{1}\right)$ is connected since $p_{1}$ is not a cut vertex in $B_{4}(I)$ and $\mathrm{SE}\left(p_{1}\right)$ has at least one black 4-neighbour. To see that $W_{4}\left(I^{1}\right)$ is connected, observe that $W_{4}(I) \backslash \mathrm{SE}\left(p_{1}\right)$ has at most two components one containing $\mathrm{s}\left(p_{1}\right)$ (if $\mathrm{s}\left(p_{1}\right)$ is white) and the other containing $\mathrm{E}\left(p_{1}\right)$ (if $\mathrm{E}\left(p_{1}\right)$ is white). Therefore, to see that $W_{4}\left(I^{1}\right)$ is connected it is enough to observe that $W_{4}\left(I^{1}\right)$ can be obtained by adding $p_{1}$ to $W_{4}(I) \backslash \operatorname{SE}\left(p_{1}\right)$ where $p_{1}$ is adjacent in $W_{4}\left(I^{1}\right)$ to at least one vertex of the first component, in particular $\mathrm{s}\left(p_{1}\right)$, and at least one vertex of the second component, in particular $\mathrm{E}\left(p_{1}\right)$. Now, in $I^{1}, p_{2}$ is an elbow but not a cut elbow anymore. Thus in $I_{1}$ elbow $p_{2}$ plays the role $p_{1}$ played in $I$. Therefore, by an easy induction (on $k$ ) we get that $I$ admits a $k$-diagonal interchange at $p$ of the second type. Note that none of the above interchanges can increase the width of the final image. That completes the proof.

To state the next lemma we need the following simple definitions. The frontier of an image $I$ is the set of all pixels in $I$ that have x-coordinate equal to the maximum $\mathrm{x}$-coordinate of the black pixels in 
$I$. Note that each image has at least one elbow in its frontier. We call the elbow in the frontier that has the maximum y-coordinate the lead elbow. An anchor of a non-vertical image $I$ is a black pixel that has the minimum $y$-coordinate amongst the black pixels that are not in the frontier, but have a (not necessarily black) 4-neighbour in the frontier (that is, an anchor is a black pixel with the minimum y-coordinate amongst all the black pixels immediately to the left of the frontier). The height of the lead elbow in a non-vertical image $I$ is defined as the difference between the y-coordinates of the lead elbow and the anchor of $I$. Note that this height may be a negative number.

Lemma 6. Any $\mathrm{B}_{4}, \mathrm{~W}_{4}$-connected non-vertical binary image I with $n$ black pixels admits a sequence of $O(n)$ 8-local interchanges, none involving the anchor of $I$, such that in the final image $J$

1. the width of $J$ is smaller than the width of $I$, or

2. the widths are the same, but the number of elbows in the frontier of $J$ is smaller than that in $I$, or

3. both quantities above are the same, but the height of the lead elbow in $J$ is greater than that in I; and, I and $J$ have the same anchor.

Proof. In an image $Q$, let $l_{Q}$ denote the lead elbow of $Q$ and let $t_{Q}$ denote the top pixel of $Q$ defined as the black pixel with the maximum y-coordinate in the frontier. Notice that the anchor of $I$ is in $\mathrm{WS}^{*}\left(t_{I}\right)$. In the proof below, for brevity, we will just state where the anchor is with respect to the pixels involved in interchanges. From that it will always be clear that no interchange involves the anchor and that in fact the anchor of each produced non-vertical image is exactly the same pixel.

Assume first that $\mathrm{NW}\left(t_{I}\right)$ is white and $\mathrm{NNW}\left(t_{I}\right)$ is black. We claim that $O(n)$ interchanges, none involving the anchor, can convert $I$ into an image $I^{\prime}$ where it is not the case that $\mathrm{NW}\left(t_{I^{\prime}}\right)$ is white and $\mathrm{NNW}\left(t_{I^{\prime}}\right)$ is black. Moreover, all these interchanges are amongst pixels in $\mathrm{N}^{+} \mathrm{W}^{*}\left(t_{I}\right)$, and the invariants are maintained, that is, the width of $I^{\prime}$ is at most that of $I$, the number of elbows in their frontiers, as well as the height of the lead elbows in the two images are the same.

If it is not the case that $\mathrm{NW}\left(t_{I}\right)$ is white and $\mathrm{NNW}\left(t_{I}\right)$ is black, then let $I^{\prime}=I$. Otherwise, $\operatorname{NNW}\left(t_{I}\right)$ is an elbow in $I$. Applying (a diagonal interchange of) Lemma 5 to $\mathrm{NNW}\left(t_{I}\right)$ gives an image $I_{1}$ where either $\operatorname{NNW}\left(t_{I_{1}}\right)$ is white, or $\operatorname{NNW}\left(t_{I_{1}}\right)$ is an elbow but not a cut elbow in $I_{1}$. If $\operatorname{NNW}\left(t_{I_{1}}\right)$ is white then let $I^{\prime}=I$, otherwise the interchange $\left\langle\mathrm{NNW}\left(t_{I_{1}}\right), \mathrm{N}\left(t_{I_{1}}\right)\right\rangle$ preserves the connectivity of the resulting image $I_{2}$. Now, in $I_{2}$, $\mathrm{NW}\left(t_{I_{2}}\right)$ is white. If $\mathrm{NNW}\left(t_{I_{2}}\right)$ is white, then let $I^{\prime}=I_{2}$, otherwise we can repeat the process above (starting by applying Lemma 5 to $\mathrm{NNW}\left(t_{I_{2}}\right)$ ) until we arrive at an image $I^{\prime}$ where it is not the case that $\mathrm{NW}\left(t_{I^{\prime}}\right)$ is white and NNW $\left(t_{I^{\prime}}\right)$ is black. That has to happen by the finiteness. None of the interchanges involves the anchor and the invariants are maintained. Furthermore, diagonal interchanges of Lemma 5 are always applied to a black pixel with bigger $y$-coordinate than in the previous iteration thus no interchange involves the same pixel. Thus the number of interchanges needed to convert $I$ to $I^{\prime}$ is at most the number of black pixels in $\mathrm{N}^{+} \mathrm{W}^{*}\left(t_{I}\right)$.

The above conversion allows us to now assume that we have an image $I$ where it is not the case that $\mathrm{NW}\left(t_{I}\right)$ is white and $\mathrm{NNW}\left(t_{I}\right)$ is black. That property is very useful, since changing the colour of $\mathrm{N}\left(t_{I}\right)$ to black results in an image that is $\mathrm{B}_{4}, \mathrm{~W}_{4}$-connected. There are two cases to consider depending on whether $l_{I}$ is a cut elbow in $I$.

Case 1: $l_{I}$ is an elbow but not a cut elbow in $I$. Let $p=l_{I}$ and let $k$ be the difference between the $\mathrm{y}$-coordinate of $t_{I}$ and the $\mathrm{y}$-coordinate of $p$. There are two sub-cases to consider here depending on whether $k$ is zero or a positive integer.

Case $1 a: k>0$. In this case, apply the following sequence of interchanges $\left(\langle p, \mathrm{NE}(p)\rangle,\left\langle\mathrm{N}^{i} \mathrm{E}(p), \mathrm{N}^{i+1} \mathrm{E}(p)\right\rangle\right.$, $\left\langle\mathrm{N}^{k} \mathrm{E}(p), \mathrm{N}^{k+1}(p)\right\rangle, 1 \leq i \leq k-1$. This is a simple set of interchanges that can be visualized as having the 
black pixel at $p$ slide upward along the east side of the frontier ending up at the top of $t_{I}$. The fact that connectivity of each resulting image $I^{1}, I^{2} \ldots I^{k+1}=J$ is preserved follows from the fact that $l_{I}$ is not a cut vertex and from the fact that it is not the case that $\mathrm{NW}\left(t_{I}\right)$ is white and $\mathrm{NNW}\left(t_{I}\right)$ is black (that, as noted above, allows us to place a black pixel on top of $\left.t_{I}\right)$. Note that the number of interchanges is at most $O(n)$ and none of them involves the anchor nor changes the anchor of the resulting image. Furthermore, the width of $J$ is at most that of $I$, the number of elbows is the same, but the lead elbow in $J$, that is $\mathrm{N}(p)$, has greater height than the lead elbow in $I$, that is $p$.

Case $1 b: k=0$. In this case each pixel in $\mathrm{N}^{+}(p)$ is white and $\mathrm{w}(p)$ is black as otherwise $p$ would be an isolated vertex. Furthermore, by the initial conversion it is not the case that $\operatorname{NW}(p)$ is white and $\operatorname{NNW}(p)$ is black, that is, either $\mathrm{NW}(p)$ is black, or both $\mathrm{NW}(p)$ and $\mathrm{NNW}(p)$ are white. Thus consider these two possibilities.

If $\mathrm{NW}(p)$ is black then the interchange $\langle p, \mathrm{~N}(p)\rangle$ preserves the connectivity and in the resulting image $J$ the width and the number of elbows in $I$ and $J$ are the same, but the lead elbow in $J$, that is $\mathrm{N}(p)$ has greater height than the lead elbow in $I$.

Thus assume $\operatorname{NW}(p)$ and $\operatorname{NNW}(p)$ are both white. Unless, $\operatorname{NNWW}(p)$ is black and $\operatorname{NwW}(p)$ is white, the interchange $\langle p, \mathrm{NW}(p)\rangle$ preserves the connectivity of the resulting image which has either the width or the number of elbows in its frontier smaller than that in $I$. Thus assume $\operatorname{NNWW}(p)$ is black and $\operatorname{Nww}(p)$ is white. Then $q=\operatorname{NNwW}(p)$ is an elbow in $I$. Applying (a diagonal interchange of) Lemma 5 to $q$ results in an image $I^{*}$ where $q$ is either white or it is an elbow but not a cut elbow in $I^{*}$. If $q$ is white then as above the interchange $\langle p, \mathrm{NW}(p)\rangle$ gives the desired result. Otherwise, the interchange $\langle q, \operatorname{SE}(q)\rangle$ followed by $\langle p, \mathrm{~N}(p)\rangle$ preserves the connectivity of both resulting images. Furthermore, the width of the final image $J$ is at most that of $I$, the number of elbows is the same but the lead elbow in $J$, that is $\mathrm{N}(p)$, has greater height than the lead elbow in $I$, that is $p$. The total number of interchanges is $O(n)$.

Case $2: l_{I}$ is a cut elbow in $I$. Again let $p=l_{I}$. Since $p$ is a cut elbow, $\mathrm{N}(p)$ and $\mathrm{w}(p)$ are black and $\mathrm{NW}(p)$ is white. Having $\mathrm{W}(p)$ black, implies that the anchor is in $\mathrm{WS}^{*}(p)$.

Now apply (a diagonal interchange of) Lemma 5 to $p$. None of the interchanges involves nor changes the anchor. In the resulting image $I^{*}, p$ is either white or it is the lead elbow that is not a cut elbow in $I^{*}$. If it is white, then we are done, namely $I^{*}=J$; the width of $J$ is at most that of $I$; the number of elbows is the same but the lead elbow in $J$, that is $\mathrm{N}(p)$, has greater height than the lead elbow in $I$, that is $p$. Otherwise if, $p$ is the lead elbow that is not a cut elbow in $I^{*}$ then we are in case $1 a$ that has already been considered.

Aided by the previous lemma we can now deduce the following theorem.

Theorem 4. Any two $\mathrm{B}_{4}, \mathrm{~W}_{4}$-connected images $I$ and $J$ each having $n$ black pixels are $(4,4)$-IP-equivalent and $I$ can be converted in $J$ using a sequence of $O\left(n^{4}\right)$ 8-local interchanges.

Proof. It is sufficient to prove that each non-vertical image $I$ with $n$ black pixels can be converted into a vertical image $J$ with $n$ black pixels, using a sequence of $O\left(n^{4}\right)$ 8-local interchanges.

Consider a sequence of images $I_{0}=I, I_{1}, I_{2}, \ldots I_{s}$ resulting from consecutive applications of Lemma 6 , such that each image in the sequence has the same width and the same number of elbows in their frontier. By Lemma 6 each image $I_{i}, 1<i \leq s$ in this sequence has the same anchor as image $I_{i-1}$, but the height of its lead elbow is greater than that in $I_{i-1}$. Therefore, there are at most $n-1$ images in this sequence, that is $s \leq n-1$. Thus applying Lemma 6 to $I_{s}$ results in an image $I_{s+1}$ that has either width or the number of elbows in its frontier smaller than $I_{s}$. Thus after at most $O\left(n^{2}\right)$ interchanges the number of elbows in the frontier goes down by one, which further implies that after at most $O\left(n^{3}\right)$ interchanges the width goes down 
by one. Thus finally, at most $O\left(n^{4}\right)$ interchanges converts $I$ into an image that has width one, that is, into a vertical image $J$.

\section{Conclusions}

We have shown that, for any $(a, b) \in\{(4,8),(8,4),(8,8)\}$, any two $\mathrm{B}_{a}, \mathrm{~W}_{b}$-connected images $I$ and $J$ each with $n$ black pixels differ by a sequence of $O\left(n^{2}\right)$ interchanges. That is the best possible, since converting a horizontal image to a vertical image requires $\Omega\left(n^{2}\right)$ interchanges. We have also shown that any two $\mathrm{B}_{4}, \mathrm{~W}_{4^{-}}$ connected images $I$ and $J$ each with $n$ black pixels differ by a sequence of $O\left(n^{4}\right)$ interchanges. Since the same $\Omega\left(n^{2}\right)$ lower bound applies to this version, the obvious open problem is whether any two $\mathrm{B}_{4}, \mathrm{~W}_{4}$-connected images differ by a sequence of $o\left(n^{4}\right)$ interchanges.

\section{Acknowledgements}

This research was initiated at the Bellairs Workshop on Computational Geometry for Folding and Unfolding, February 2nd to 8th, 2002. The authors are grateful to Godfried Toussaint for organizing the workshop and to the other workshop participants for providing a stimulating working environment.

\section{References}

[1] A. Dumitrescu and J. Pach. Pushing squares around. In Proceedings of the 20th Annual ACM Symposium on Computational Geometry (SoCG2004), pages 116-123, 2004.

[2] T. Y. Hong and A. Rosenfeld, editors. Topological Algorithms for Digital Image Processing. Elsevier, Amsterdam, 1996.

[3] T. Y. Kong and A. Rosenfeld. Digital topology: Introduction and survey. Computer Vision, Graphics and Image Processing, 48:357-393, 1989.

[4] A. Rosenfeld. Connectivity in digital pictures. Journal of the ACM, 17(1):146-160, 1970.

[5] A. Rosenfeld. Arcs and curves in digital pictures. Journal of the ACM, 20(1):81-87, 1973.

[6] A. Rosenfeld. Adjacency in digital pictures. Information and Control, 26:24-33, 1974.

[7] A. Rosenfeld, T. Y. Kong, and A. Nakamura. Topology-preserving deformations of two-valued digital pictures. Graphical Models and Image Processing, 60:24-34, 1998.

[8] A. Rosenfeld and A. Nakamura. Two simply connected sets that have the same area are IP-equivalent. Pattern Recognition, 35(2):537-541, 2002.

[9] A. Rosenfeld, P. K. Sala, and A. Nakamura. Interchangeable pairs of pixels in digital images. Pattern Recognition, 34(9):1853-1865, 2001. 